Journal of Engineering and Applied Sciences 14 (Special Issue 6): 9519-9522, 2019

ISSN: 1816-949X

(C) Medwell Journals, 2019

\title{
Preparation of Porous Silicon Photodetector Enhanced by Ag Nanoparticle
}

\author{
Ahmed S. Ahmed \\ Department of Medical Science, College of Nursing, University of Misan, Amarah, Iraq
}

\begin{abstract}
This research investigation the effect of Ag nanoparticle to improvement the sensitivity of photodetector. Porous silicon prepared with photoelectrochemical etching by using n-type silicon substrate. To increase the efficiency of detection deposited the Ag nanoparticle on porous silicon surface by annealing process. SEM and AFM technique were used to study the morphological characteristics of the PSi surface. Dark and photo current-voltage and responsivity were probed before and after the deposit Ag nanoparticle and the responsivity increased from $0.35-0.6 \mathrm{~A} / \mathrm{W}$.
\end{abstract}

Key words: Porous silicon, annealing, depositing, Ag nanoparticles, efficiency, photo current-voltage

\section{INTRODUCTION}

The latest notice of powerful visible photoluminescence in anodically etched porous silicon has created substantial attention (Brandt et al., 1992). Very easy factors demonstrate that two assumptions are needed for effective emission of visible light from silicon components: a confinement of providers into nanometer-sized silicon cells in purchase to bring sufficient confinement to take optical transitions in the visible array; an improvement of the light quantum effectiveness which can bring its source from an enhance in the radiative recombination rate considering of the busting of energy conservation or from a reducing of the nonradiative works by a passivation of the limited region surfaces (Vial et al., 1992) these advantage are suitable for building photodetector.

Newly optical properties have been aimed for detection, admitting variant of PL, reflectivity and optical wave guiding. Optical detectors are predicting because of their very quick response (Mulloni and Pavesi, 2000).

The porous silicon photodetectors produced by traditional methods are limited in efficiency due to the lack of stability inelectrical and optical properties, deficient dark current and deficient photo current deficient light sensitivity, so, the scope of application is limited (Ismail et al., 2017).

The porous silicon photodetector manufactured by RTO process are widely used as optical devices because of their high quality in sensitivity of light. The accelerate of a PSi photodetector is significant figure of merit ( $\mathrm{Yu}$ and Wie, 1993). There are three basic types of porous silicon photodetectors such like metal semiconductor metal, p-n junction and shottky diode were manufactured to detect light (Rahim et al., 2011).
Rossi and Bohn (2005) reported the improvement of porous silicon photodetector by using rapid thermal oxidation RTO process at $850^{\circ} \mathrm{C}$ where the responsivity was around $2.5 \mathrm{~A} / \mathrm{W}$ and raised to $5.5 \mathrm{~A} / \mathrm{W}$ at $800 \mathrm{~nm}$. Thouti et al. (2013) observed reduced reflection losses and improved absorption of light in visible region after depositing Ag nanoparticles on silicon substrate. In this research, used $\mathrm{Ag}$ nanoparticle film instead of RTO process where the responsivity is improvement after depositing on porous Silicon.

\section{MATERIALS AND METHODS}

Experiment: The porous silicon for this study created by photo electrochemical etching on n-type (100) silicon wafers $(1-3 \Omega \mathrm{cm})$. The anodization current density was $12 \mathrm{mAcm}^{-2}$ had a porosity equal to $70 \%$ and using a Teflon cell with an electrolyte containing HF: ethanol (2:1). For photo electrochemical etching using a laser with the wavelength $560 \mathrm{~nm}$. The morphological study of porous silicon was started by SEM and AFM before and after adding Ag nanoparticle. The porosity of the porous silicon as determined gravimetrically was $60 \%$.

Figure 1 shows the schematic diagram of photoelectrochemical etching used for porous silicon formation. To enhance the electrical properties was made on back surface by depositing aluminum with a thickness $100 \mathrm{~nm}$ by annealing under a vacuum at $350^{\circ} \mathrm{C}$ for $20 \mathrm{~min}$. The electrical properties (dark and illuminated I-V) of photodetector were studied in laboratories temperature by using electro meter and two light intensity.

The deposited silver film were afterward annealed at $450^{\circ} \mathrm{C}$ for $45 \mathrm{~min}$ in nitrogen situation to alter the surface structure. The thickness of Ag thin film was $100 \mathrm{~nm}$ 


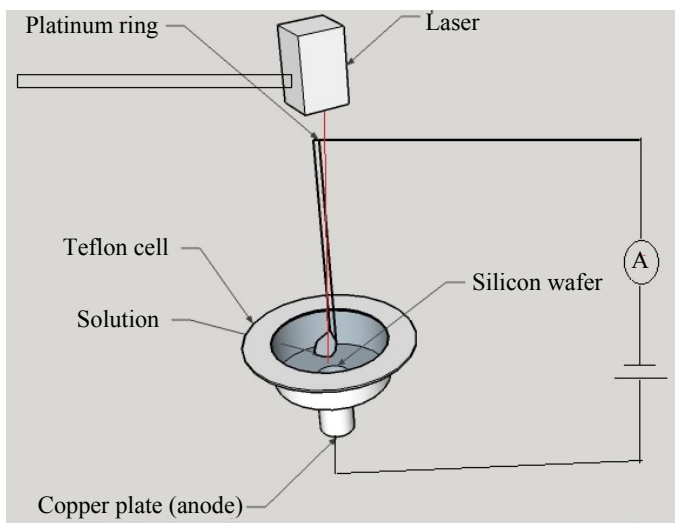

Fig. 1: Schematic diagram of photo electrochemical etching

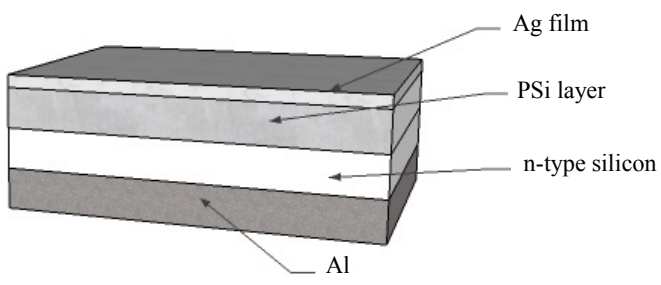

Fig. 2: Cross-section of $\mathrm{Ag} / \mathrm{PSi} / \mathrm{Si} / \mathrm{Al}$ photodetector

where this thickness was suitable for this photodetector. Figure 2 shows the cross-sectional of the $\mathrm{Ag} / \mathrm{PSi} / \mathrm{Si} / \mathrm{Al}$ structure.

\section{RESULTS AND DISCUSSION}

The SEM images of the silicon pore layer show a coarse surface structure with a number of nanosized pores as shown in Fig. 3. The pores contain cylindrical and rectangular shapes that are randomly distributed, vertically perpendicular to the sample level where the porous diameters range from $0.3-1.5 \mu \mathrm{m}$. The increase in pore diameter is due to increase in the number of holes on silicon surface when the laser light is absorbed by the upper layer of silicon.

The SEM images showed some variation in porous silicon morphology before and after the deposition of silver particles. Figure 4 shows SEM image of Ag films deposited on porous silicon by annealing at $450^{\circ} \mathrm{C}$ for $45 \mathrm{~min}$ in vacuum environment. The deposited nanoparticles were in the form of small sizes distributed on top and also with large nanoparticles size. The nanoparticles fill holes located on porous silicon surface where the image of the electron microscope shows how these holes are filled.

The AFM images of the porous silicon layer before and after addition of nanoparticles for silver are shown in Fig. 5 where a change in silicon surface is observed in
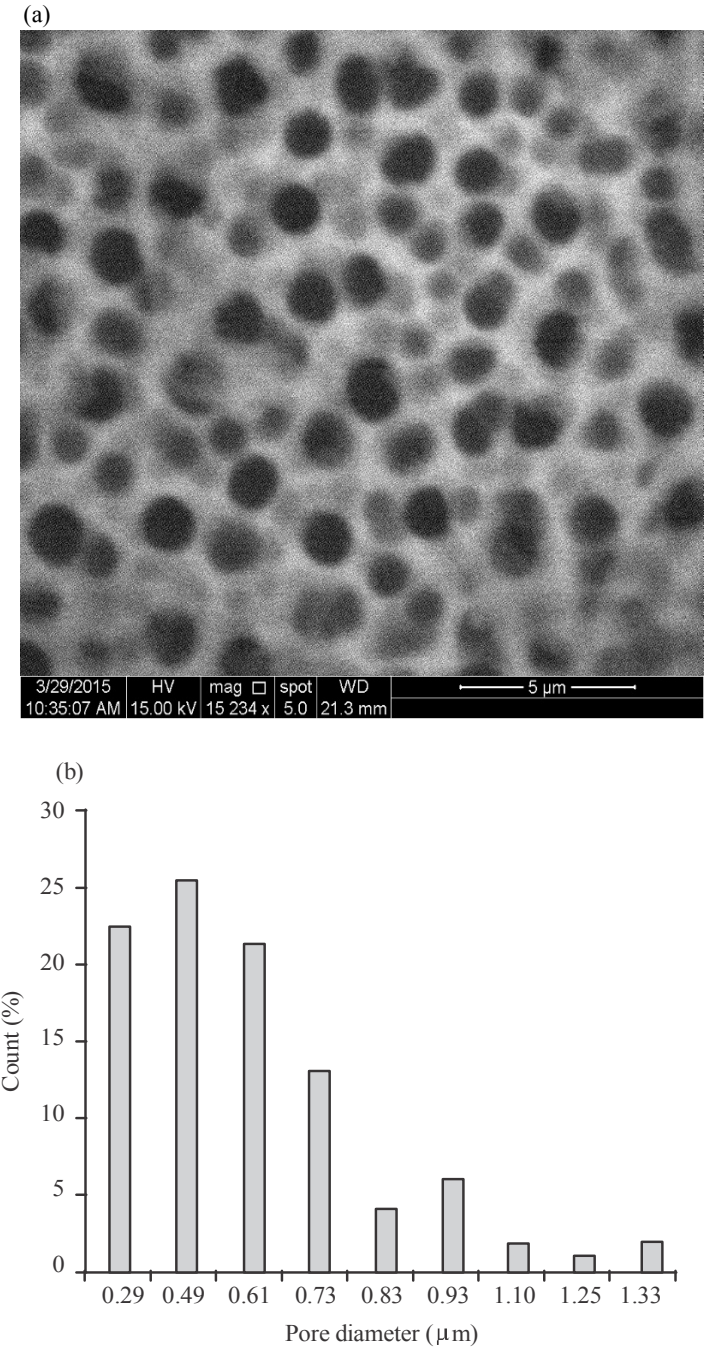

Fig. 3: a, b) SEM image for fresh PSi

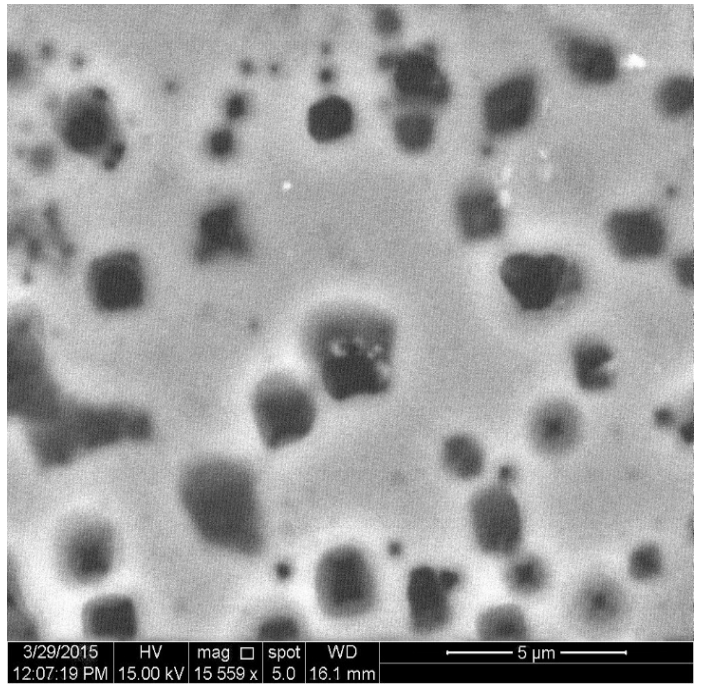

Fig. 4: SEM image after adding Ag nanoparticle 

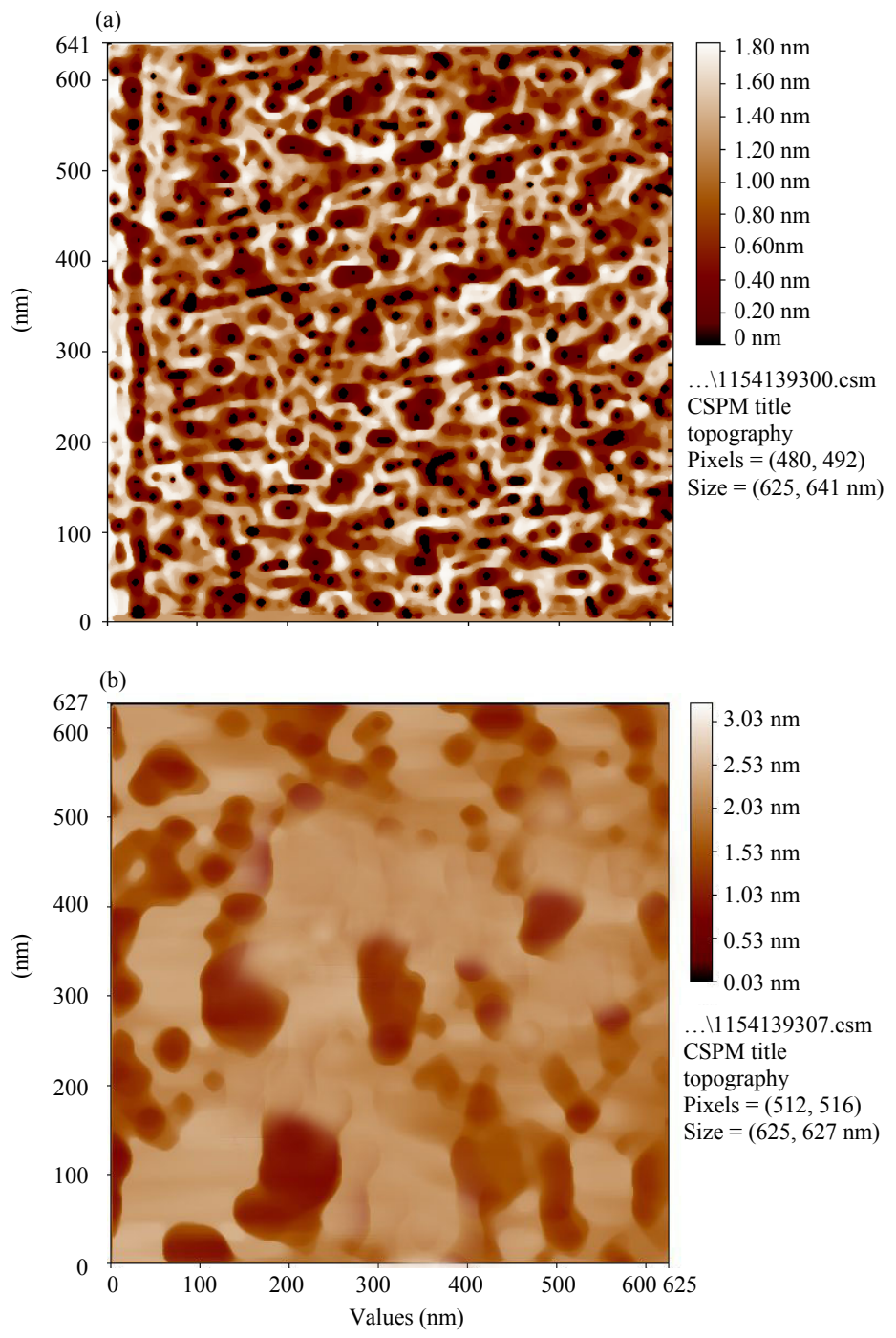

Fig. 5: 2D AFM image: (a) Fresh porous silicon and (b) After adding Ag nanoparticle

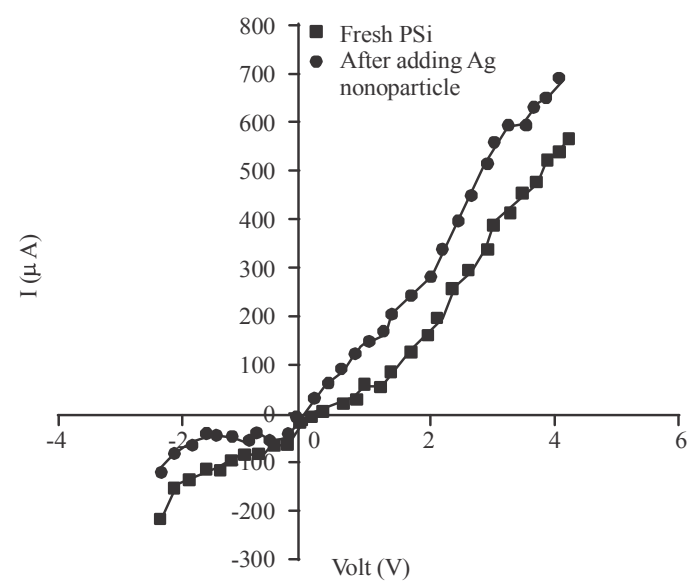

Fig. 6: Behavior of I-V characteristics before and after adding Ag nanoparticle in dark after adding silver particles where the boundaries of the pores were clear and the thickness of the wall of the pores increased also after the addition of these nanoparticles.

Figure 6 shows the behavior of the dark current before and after the addition of silver nanoparticles in the forward and reverse bias where the structure $(\mathrm{Ag} / \mathrm{PSi} / \mathrm{Si} / \mathrm{Al}$ ) possesses $\mathrm{Ag} / \mathrm{PSi}$ Schottky and $\mathrm{Si} / \mathrm{Al}$ heterojunction.

The addition of silver particles to the porous silicon surface causes an increase in the forward current and reduces the resistance of porous silicon layer and the reverse current decreases after the addition.

Another important way to determine the electrical properties of PS is current measurements under lighting. Figure 7 display the $\mathrm{I}_{\mathrm{PH}^{-}}-\mathrm{V}$ characteristics of porous silicon as a photodetector after illuminated under light intensity 


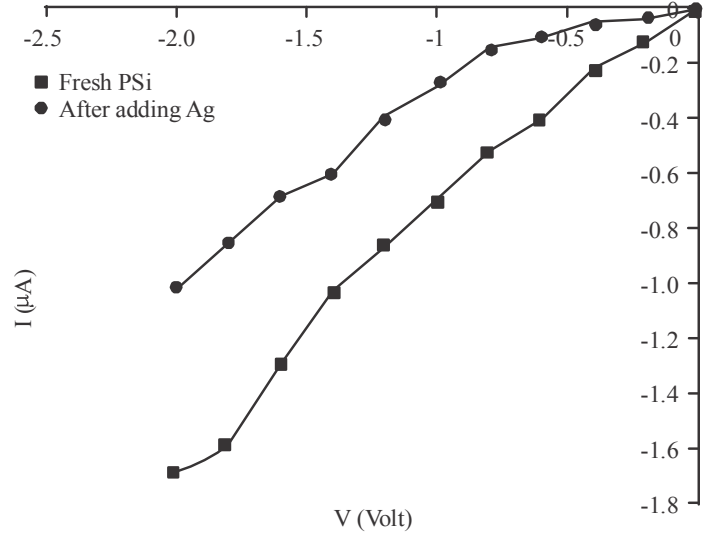

Fig. 7: Behavior of I-V characteristics before and after depositing Ag nanoparticle in illumination

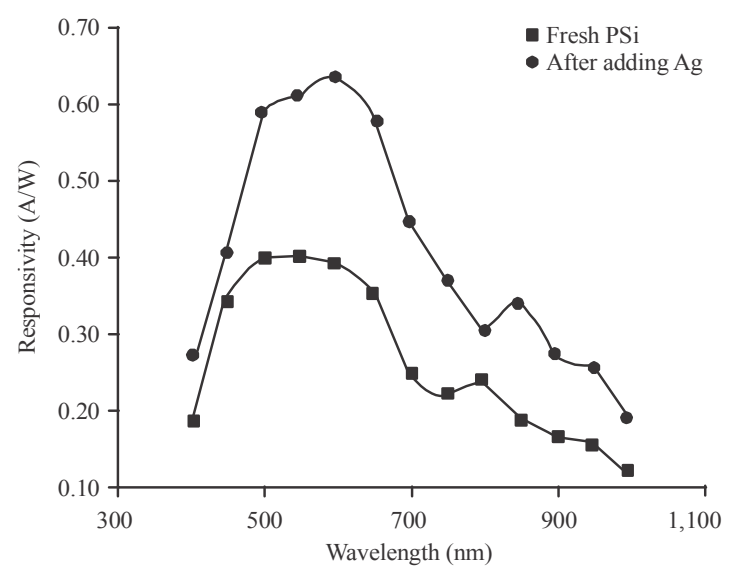

Fig. 8: Responsivity before and after adding Ag nanoparticle

before and after adding $\mathrm{Ag}$ nanoparticles where the increase in current from $0.8-1.6 \mathrm{~mA}$ at $2 \mathrm{~V}$ is observed compared to before adding silver nanoparticles. This improvement can be attributed to increased absorption in the surface of the porous and also short the length of diffusion of photo generated carriers as well as by increasing photo receptors carrier from silver particles to porous silicon. The photo responsivity of PSi before and after adding $\mathrm{Ag}$ nanoparticle are shown in Fig. 8.
Where the response was between 400 and $1000 \mathrm{~nm}$ in wavelengths. The maximum photoresponse was $0.6 \mathrm{~A} / \mathrm{W}$ in $695 \mathrm{~nm}$ due to raising absorption of light by $\mathrm{Ag}$ nanoparticle where the responsivity after adding $\mathrm{Ag}$ nanoparticle was better than from fresh PSi.

\section{CONCLUSION}

In this research, we prepared a photodetector with low dark current in reverse bias by depositing $\mathrm{Ag}$ nanoparticle and with increasing in photocurrent after depositing by annealing process where the photocurrent increased from $0.8-1.8 \mathrm{~mA}$ at $2 \mathrm{~V}$. The responsivity was also, enhanced after $\mathrm{Ag}$ nanoparticle deposited and we get the best response in $0.6 \mathrm{~A} / \mathrm{W}$ under light illumination.

\section{REFERENCES}

Brandt, M.S., H.D. Fuchs, M. Stutzmann, J. Weber and M. Cardona, 1992. The origin of visible luminescencefrom porous silicon: A new interpretation. Solid State Commun., 81: 307-312.

Ismail, R.A., A.M. Alwan and A.S. Ahmed, 2017. Preparation and characteristics study of nano-porous silicon UV photodetector. Appl. Nanosci., 7: 9-15.

Mulloni, V. and L. Pavesi, 2000. Porous silicon microcavities as optical chemical sensors. Appl. Phys. Lett., 76: 2523-2525.

Rahim, A.F.A., M.R. Hashim and N.K., 2011. High sensitivity of palladium on porous silicon MSM photodetector. Phys. B. Condens. Matter, 406: 1034-1037.

Rossi, A.M. and H.G. Bohn, 2005. Photodetectors from porous silicon. Phys. Status Solidi A., 202: 1644-1647.

Thouti, E., N. Chander, V. Dutta and V.K. Komarala, 2013. Optical properties of Ag nanoparticle layers deposited on Silicon substrates. J. Opt., 15: 1-7.

Vial, J.C., A. Bsiesy, F. Gaspard, R. Herino and M. Ligeon et al., 1992. Mechanisms of visible-light emission from electro-oxidized porous silicon. Phys. Rev. B., 45: 14171-14176.

Yu, L.Z. and C.R. Wie, 1993. Study of MSM photodetector fabricated on porous silicon. Sens. Actuators A. Phys., 39: 253-257. 